\section{Viral infection: the clot thickens}

Many invertebrates coopt their coagulation cascade to resist microbial infection; however, it has been unclear whether mammals use an equivalent system. In Science, Shayakhmetov and colleagues demonstrate that the human blood-coagulation factor FX is important for the recognition of and immune response to adenovirus. After infection, FX rapidly coats the adenoviral hexon and engages Toll-like receptor 4 (TLR4) on tissue macrophages. FX therefore seems to facilitate binding in a way analogous to the well-known TLR4 coreceptor MD2. Engagement of TLR4 by FX-coated adenovirus triggers a host of inflammatory cytokines via a classic signaling pathway dependent on the adaptor MyD88 and transcription factor NF-kB, whereas mutant virus unable to bind FX does not trigger TLR4 signaling. This mechanism of FX-mediated release of inflammatory cytokines might underlie some of the adverse immunological reactions that have hampered adenoviral gene-therapy approaches.

Science 338, 795-798 (2012)

\section{Plasma-cell survival}

Long-term humoral immunity requires memory B cells and long-lived antibody-secreting cells (ASCs). In Science Signaling, van Spriel et al. show that optimal long-term immunoglobulin $\mathrm{G}$ responses require the tetraspanin protein CD37. There is abundant CD37 on the surface of mature B cells, which seems to be necessary for clustering of the integrin $\alpha_{4} \beta_{1}$, required for high-affinity binding to its ligand, VCAM-1. The VCAM-1- $\alpha_{4} \beta_{1}$ interaction triggers an outside-in signaling cascade that activates the kinase Akt, which in turn phosphorylates and inactivates the proapoptotic molecule Bad. CD37-deficient mice develop germinal centers after immunization but produce less antibody and have fewer immunoglobulin G-producing plasma cells than do wild-type mice. These data suggest CD37-induced clustering of $\alpha_{4} \beta_{1}$ contributes to the long-term survival of plasma cells.

Sci. Signal. (13 November 2012) doi:10.1126/scisignal.2003113

\section{It takes two}

Expression of the transcription factor Foxp3 is essential but might not be sufficient for the development of regulatory $T$ cells ( $\mathrm{T}_{\text {reg }}$ cells). In Immunity, Sakaguchi and colleagues show that hypomethylation of certain $\mathrm{T}_{\text {reg }}$ cell-representative gene regions is required for the development of natural $\mathrm{T}_{\text {reg }}$ cells ( $\mathrm{nT}_{\text {reg }}$ cells) and that it is established in the thymus independently of Foxp3 expression. CpG hypomethylation of limited regions (Foxp3 intron 1, Tnfrsf 18 exon 5, Ctla4 exon 2 and Ikzf4 intron 1b) is exclusively 'imprinted' in $\mathrm{nT}_{\text {reg }}$ cells and not in other $\mathrm{T}$ cell populations, including inducible $\mathrm{T}_{\text {reg }}$ cells. Hypomethylation of the $\mathrm{nT}_{\text {reg }}$ cell type is required for Foxp $3^{+} T$ cells to acquire $n T_{\text {reg }}$ cell-type gene expression and full suppressive activity and to sustain the expression of $T_{\text {reg }}$ cell-associated molecules such as Foxp3, CLTA-4 and CD25. The induction of such hypomethylation in developing $\mathrm{nT}_{\text {reg }}$ cells is dependent on engagement of the TCR by self ligands in the thymus.

Immunity 37, 785-799 (2012)

\section{Activation by calcium}

Several models of inflammasome activation, such as $\mathrm{K}^{+}$efflux, generation of reactive oxygen species and lysosomal destabilization, have been proposed. In Nature, Chae and colleagues show that recognition of extracellular $\mathrm{Ca}^{2+}$ through the calcium-sensing receptor CASR activates the NLRP3 inflammasome. CASR activates phospholipase C, which catalyzes the production of inositol-1,4,5-triphosphate and release of $\mathrm{Ca}^{2+}$ from endoplasmic reticulum stores. A greater abundance of cytoplasmic $\mathrm{Ca}^{2+}$ promotes assembly of components of the NLRP3 inflammasome. Stimulation via CASR also diminishes the abundance of intracellular cAMP, which binds NLRP3 directly to inhibit inflammasome assembly. Thus, downregulation of cAMP relieves this inhibition. Mutant NLRP3 from patients with cryopyrin-associated periodic syndrome binds cAMP with lower affinity than does wild-type NLRP3. Increasing cAMP attenuates the uncontrolled production of interleukin $1 \beta$ by peripheral blood mononuclear cells in such patients. These results suggest that extracellular $\mathrm{Ca}^{2+}$ can function as a danger signal.

Nature (11 November 2012) doi:10.1038/nature11588

\section{Building enhancers}

Cytokines provide polarizing signals that dictate the outcome of effector T cell responses. In Cell, O'Shea and colleagues characterize how STAT transcription factors contribute to chromatin remodeling associated with the polarization of $\mathrm{CD}^{+}$ $T$ cells into the $T_{H} 1$ and $T_{H} 2$ helper $T$ cell subsets. Active enhancer elements are marked by monomethylation of histone 3 at Lys4 (H3K4me1) and the histone acetyltransferase p300, which confers acetylation of $\mathrm{H} 3$ at Lys27. Genome-wide profiling shows that deposition of $\mathrm{H} 3 \mathrm{~K} 4 \mathrm{me} 1$ at enhancer elements does not discriminate $T_{H} 1$ and $T_{H} 2$ cells. Instead, STAT proteins occupy lineage-specific enhancer sites together with $\mathrm{p} 300$. Such binding of p300 is lost in STAT-deficient T cells and, concomitantly, their expression of lineage-appropriate gene is also lower. Surprisingly, the $T_{H} 1$-specific transcription factor T-bet does not substantially alter the p300-H3K4me1 landscape; instead, T-bet is necessary for the suppression of alternative geneexpression programs. These findings suggest that STAT proteins are needed to recruit chromatin-remodeling enzymes to activate enhancers and direct lineage-appropriate gene expression. $L A D$ Cell 151, 981-993 (2012)

\section{Mimicking nickel allergy}

An allergy to nickel $\left(\mathrm{Ni}^{2+}\right)$ is one of the most common forms of contact dermatitis, but how $\mathrm{T}$ cells recognize $\mathrm{Ni}^{2+}$-haptenized peptides is unclear. In the Proceedings of the National Academy of Sciences, Dai and colleagues use structural insights obtained with $\mathrm{ANi} 2.3$, a Ni${ }^{2+}$-reactive $\mathrm{T}$ cell antigen receptor (TCR), to shed light on the basis of $\mathrm{Ni}^{2+}$ allergy. ANi2.3 recognizes an unknown $\mathrm{Ni}^{2+}$-haptenized peptide in the context of HLA-DR52c. Their screen fails to identify the $\mathrm{Ni}^{2+}$-haptenized peptide recognized by ANi2.3, probably because of low abundance of the natural ligand. However, it identifies many 'mimetopes' recognized by ANi2.3 in the absence of $\mathrm{Ni}^{2+}$. Structural and mutational analysis of the mimetope-DR52c complex shows a highly conserved lysine at peptide position 7 that is essential for recognition by ANi2.3. The $\varepsilon$-amino group of this lysine interacts with complementarity-determining region 3 of ANi2.3 in a manner analogous to $\mathrm{Ni}^{2+}$, most probably because of its equivalent positive side chains.

Proc. Natl. Acad. Sci. USA 109, 18517-18522 (2012) 